\title{
Comparison of new immunohistochemical assay for oestrogen receptor in paraffin wax embedded breast carcinoma tissue with quantitative enzyme immunoassay
}

\author{
G Saccani Jotti, S R D Johnston, J Salter, S Detre, M Dowsett
}

\begin{abstract}
Aim-To validate the use of a new mouse monoclonal antibody (1D5) directed against the $\mathbf{N}$-terminal domain (A/B region) of the oestrogen receptor in an immunohistochemical assay (ER-IHA) for paraffin wax embedded tissue.

Methods-Breast cancer specimens were surgically obtained from 119 previously untreated patients. For comparison, oestrogen receptor was measured from cytosol fractions using an established oestrogen receptor enzyme immunoassay (ER-EIA) method. Oestrogen receptor "H-scores" were obtained from the ERIHA after antigen retrieval using microwave treatment. Where discrepancies occurred between the two methods, further immunohistochemistry was performed using the H222 antibody from the Abbott Laboratories ER-ICA kit.
\end{abstract}

Results-The correlation between the two methods was non- linear, but despite this there was an $86 \%$ concordance between ER-EIA and ER-IHA using the $1 D 5$ ER antibody. Fifty four per cent of tumours (64/119) were oestrogen receptor positive and $32 \%(38 / 119)$ were negative by both assays. A mismatch between the ER-EIA and ER-IHA occurred in 17 cases. Seven tumours were IHA positive but EIA+, but five of these were borderline negative by EIA, having values of $>5$ and $<10 \mathrm{fmol} / \mathrm{mg}$ protein. Ten tumours were IHA negative and EIAt; four of these tumours were completely negative by IHA in the section studied. A further IHA assay, carried out on the 17 tumour mismatches with H222 antibody, showed that three tumours remained substantially discordant. These three tumours were strongly positive with the $1 \mathrm{D5}$ antibody and negative with the H222 antibody. Two of these discordant tumours were of the rare ER negative and PgR positive phenotype and may contain oestrogen receptor that is of biological interest but which lacks the hormone binding epitope.

Conclusions - The concordance between the classic enzyme immunoassay technique and the new immunohistochemical method on paraffin wax embedded sections was good. Moreover, the IHA technique using the 1D5 antibody against the $\mathbf{N}$-terminal was easily reproducible. This technique may allow oestrogen receptor content to be determined in large cohorts of patients in whom archival tumour material is available.

(F Clin Pathol 1994;47:900-905)

Oestrogen receptor is the biochemical marker most frequently measured in breast cancer. The presence of oestrogen receptors in breast cancer is an indicator of good prognosis, ${ }^{12}$ and tumours rich in oestrogen receptor have a 60 to $70 \%$ chance of responding to first line endocrine treatment. ${ }^{3}$ As a result of this, the choice of treatment for individual patients may be influenced by oestrogen receptor content. New methods of measurement must therefore be subject to close scrutiny and optimisation.

The monoclonal antibody $\mathrm{H} 222$, which is directed against the hormone binding region close to the C-terminal end of the human oestrogen receptor, ${ }^{4}$ has been used to quantify the expression of oestrogen receptor in homogenates of breast cancer by immunochemical means using a sandwich enzyme immunoassay technique (ER-EIA). ${ }^{5}$ This enzyme immunoassay has increasingly superseded the ligand binding assay (ER-DCC) because of its convenience and superior reproducibility between and within laboratories. ${ }^{6}$ In recent years it has tended to be the yardstick against which evolving methodologies have been compared. ${ }^{7}$ When clinical specimens are too small for biochemical assay, the $\mathrm{H} 222$ antibody has been used to probe frozen tissue sections in an immunocytochemical assay (ER-ICA). This approach allows the normal and benign positive staining elements in tumours to be excluded from assessment, a potential source of false positive results in biochemical assays. ER-ICA assays can be cost effective and quick to perform, although until recently have been applicable only to frozen tissue sections.

The availability of a reliable immunohistochemical assay on paraffin wax embedded tumours (ER-IHA) would be of great value in studies of archival material. Antigen retrieval by enzyme predigestion of tissue sections has made oestrogen receptor determination technically possible in paraffin wax embedded breast cancers which have been fixed in formalin. ${ }^{8}$ The conditions required for antigen retrieval depend on the fixative, fixation period, and the processing temperature. ${ }^{9}$ The 
use of the $\mathrm{H} 222$ antibody in this context has met with varying degrees of reported success, and inconsistencies between and within laboratories have been common. ${ }^{10}$ The lack of consistency could be due to differing pronase activity between batches, although with optimisation of predigestion techniques, individual laboratories have got the $\mathrm{H} 222$ antibody to work well and consistently.

Because of these problems, however, we investigated microwave pretreatment as an alternative and more reliable method of antigen retrieval, in addition to using a new antioestrogen receptor antibody (clone 1D5), directed against the $\mathrm{N}$-terminal region of the oestrogen receptor (Dako), ${ }^{11}$ which is considerably cheaper than $\mathrm{H} 222$.

\section{Methods}

The study was carried out on 119 untreated primary breast carcinomas. Mastectomy or excision biopsy specimens were delivered fresh immediately after resection, and were processed immediately.

A portion of tumour, about $200 \mathrm{mg}$ in size, was snap frozen in liquid nitrogen immediately after dissection from the breast, and stored at $-80^{\circ} \mathrm{C}$ before analysis for oestrogen receptor using enzyme immunoassay. The remainder of the tumour was fixed for about 24 hours in $10 \%$ buffered formalin. The tissues were embedded in paraffin wax following a routine processing procedure which did not exceed $60^{\circ} \mathrm{C}$. As far as possible, the portion of tumour taken for ER-EIA was immediately adjacent to the paraffin wax embedded tumour from which sections were cut.

Sections of $3 \mu \mathrm{m}$ were cut from the paraffin wax embedded tumours on to slides coated with APES (3-aminopropyltriethoxysilane; Sigma). The sections were air dried overnight in an oven at $37^{\circ} \mathrm{C}$, and one section stained with haematoxylin and eosin for light microscopic assessment.

\section{OESTROGEN RECEPTOR ENZYME IMMUNOASSAY} (ER-EIA)

Before cytosol preparation, fat, blood, and necrotic tissue were discarded from each sample and a piece of tumour between $0 \cdot 1$ and $0.2 \mathrm{~g}$ was taken. The tissue was finely chopped, weighed, and transferred to the Teflon container of a microdismembrator (Braun Medical Ltd.). The tissue was pulverised for one minute after being cooled in liquid nitrogen for one minute. The powdered tumour was reconstituted 1:8 weight:volume in iced TRIS/molybdate buffer $(5 \mathrm{mM}$ sodium molybdate, $10 \mathrm{mM}$ monothioglycerol, $1 \mathrm{mM}$ dipotassium chloride EDTA, $3 \mathrm{mM}$

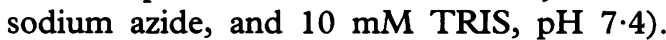
The homogenate was centrifuged at $4^{\circ} \mathrm{C}$ for 20 minutes at $2000 \times g$ after which the cytosol fraction was removed and diluted 1 in 5 in TRIS/molybdate buffer for protein assay using the Bio-Rad method with a bovine gamma globulin standard.

An aliquot of the cytosol was diluted to give a protein concentration of 1 to $2 \mathrm{mg} / \mathrm{ml}$. The oestrogen receptor concentrations in the diluted cytosols were determined using the ER-EIA kit from Abbott Diagnostics according to the manufacturer's instructions and values $\geq 10 \mathrm{fmol} / \mathrm{mg}$ protein were regarded as positive.

\section{D5 ER IMMUNOHISTOCHEMICAL METHOD} (ER-IHA-1D5)

The paraffin wax embedded sections were warmed on a hot plate and then dewaxed in xylene and rehydrated through a descending series of alcohols. Unless otherwise stated all the following reagents were obtained from Dako Laboratories and incubations carried out at room temperature. The sections were placed in a plastic dish filled with $10 \mathrm{mM}$ citrate buffer, $\mathrm{pH} 6.0$, and microwaved $(750$ watts) on full power for two five minute intervals, ensuring that the sections did not dry out at any time. The sections were then washed in tap water and phosphate buffered saline (PBS) (pH7.4) and incubated in 10\% hydrogen peroxide/PBS for 15 minutes, and a 1 in 5 dilution of normal rabbit serum for five minutes. The normal rabbit serum was tapped off and the sections were then incubated for two hours with a 1 in 100 dilution of monoclonal anti-human ER antibody 1D5. The sections were washed and incubated with a 1 in 100 dilution of biotinylated rabbit antimouse immunoglobulin for 45 minutes. After washing in PBS, the sections were incubated with a 1 in 200 dilution of horseradish peroxidase conjugated streptavidin complex for one hour. A solution of $0.05 \% 3,3^{\prime}$ diaminobenzidine (Sigma) dissolved in dimethyl formamide, plus $100 \mu \mathrm{l}$ of 30 volumes hydrogen peroxide $/ 100 \mathrm{ml}$ PBS, was used to develop the peroxidase activity in the sections for 10 minutes. The sections were then washed in running water, counterstained lightly with Mayer's haematoxylin, blued in tap water, dehydrated, cleared, and mounted. Previously identified strongly oestrogen receptor positive paraffin wax embedded tumours were used as controls, with the negative control being achieved by omission of the primary antibody.

\section{H222 ER IMMUNOHISTOCHEMICAL METHOD \\ (ER-IHA- H222)}

Serial sections of tissue adjacent to those stained with 1D5 were taken for staining with $\mathrm{H} 222$. The method was the same as for the ER-IHA-1D5 described above, with two exceptions. The sections were not microwaved, but instead, following incubation with $\mathrm{H}_{2} \mathrm{O}_{2}$, were digested with a $0.03 \%$ solution of XXV protease enzyme (Sigma) in PBS for nine minutes at $40^{\circ} \mathrm{C}$ and rinsed in PBS at $4^{\circ} \mathrm{C}$. After incubation with a 1 in 5 dilution of normal rabbit serum for five minutes, sections were incubated overnight with rat primary monoclonal anti-human oestrogen receptor antibody $\mathrm{H} 222$ (Abbott Diagnostics ER-ICA Kit). The other difference in the immunohistochemical protocol was that the incubation time with the streptavidin biotinylated horseradish peroxidase complex was half an hour shorter. 


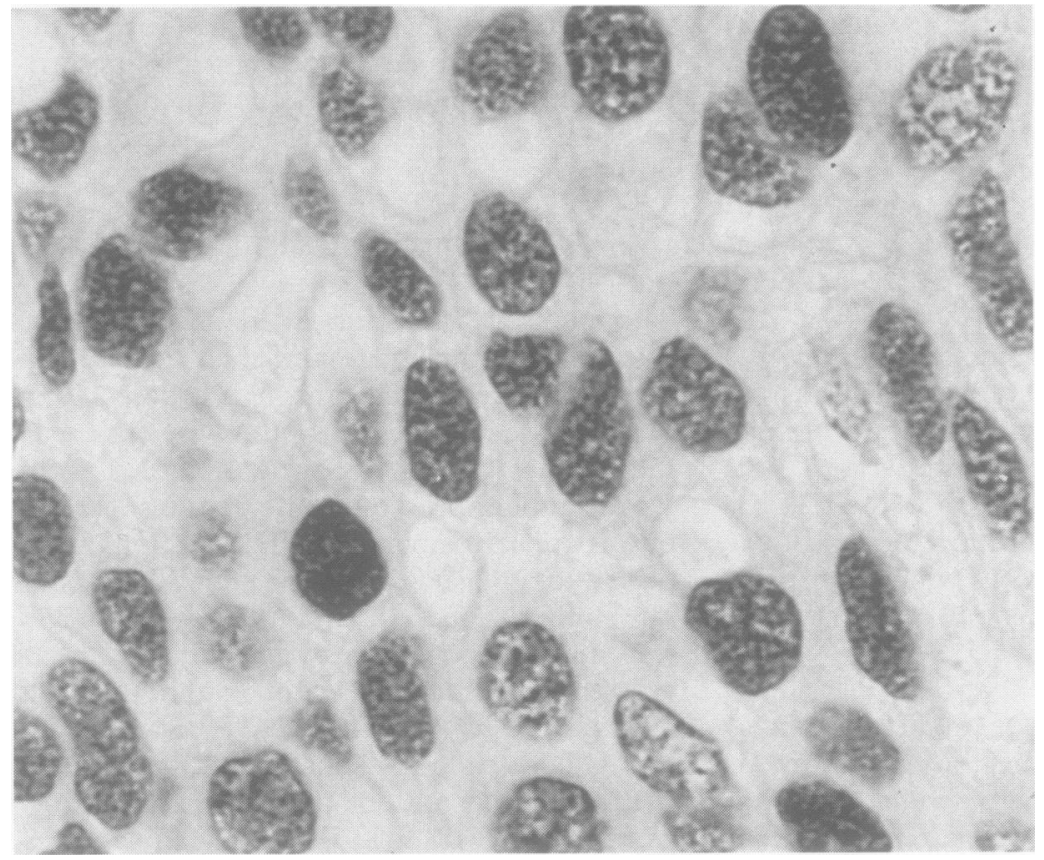

Figure 1 Paraffin wax embedded section of an invasive ductal carcinoma of the breast stained with $1 D 5$ monoclonal antibody against oestrogen receptor, detected by immunoperoxidase and conterstained with weak haematoxylin. Uniform nuclear staining is observed in tumour cells.

\section{SCORING ASSESSMENT}

The sections were assessed without knowledge of the clinical and biochemical data. In scoring the immunohistochemically stained sections 10 fields were chosen at random at $\times 400$ magnification (objective $\times$ eyepiece) and a minimum of 500 cells were counted. The intensity of staining in carcinoma cells was assessed semiquantitatively using a scoring index of 0 to 3, corresponding to negative, weak, intermediate, and strong positive staining intensity. The percentage of cells stained at each intensity was estimated. The product of the scoring index and the percentage gave an overall $\mathrm{H}$ score ranging from 0 to $300 .^{12}$ Stroma, normal, and benign epithelial tissue were excluded from this assessment. A tumour was designated oestrogen receptor positive if the $\mathrm{H}$ score was $\geq 20$.

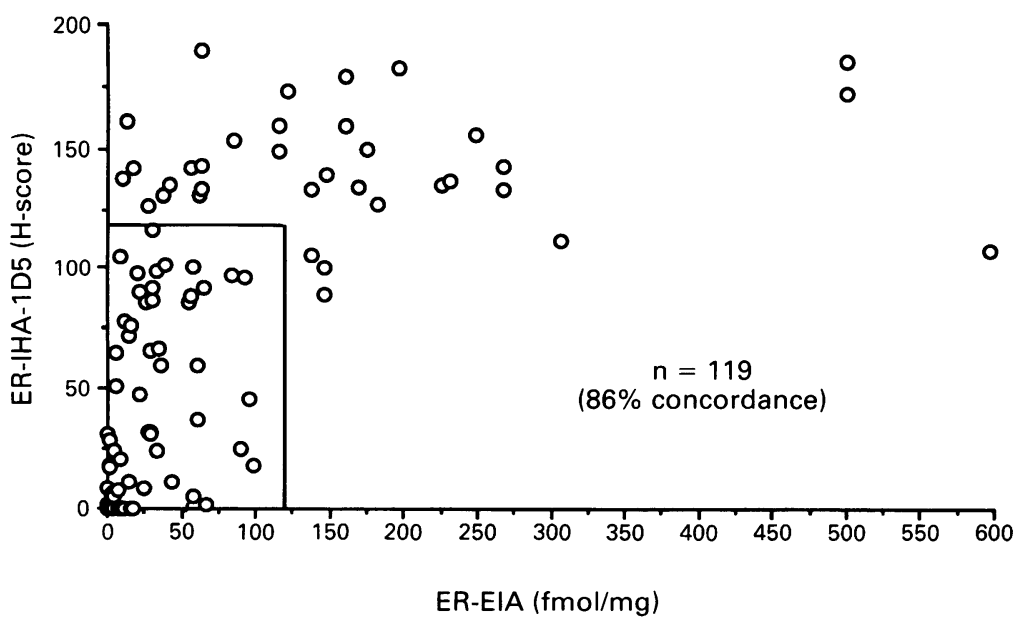

Figure 2 Correlation between quantitative ER-ELA (fmol/mg protein) and semiquantitative ER-IHA (H score) in 119 untreated primary breast cancers.
Table 1 Concordance between enzyme immunoassay (ER-EIA) and immunohistochemical assay with $1 D 5$ antibody (ER-IHA-1D5) in 119 untreated primary breast cancers

\begin{tabular}{llcc}
\hline & $E L A+$ & $E L A-$ & Totals \\
\hline IHA + & $64(54 \%)$ & $7(6 \%)$ & 71 \\
IHA- & $10(8 \%)$ & $38(32 \%)$ & 48 \\
Totals & 74 & 45 & 119 \\
\hline
\end{tabular}

$\chi^{2}=55.6$; degrees of freedom $=1 ; p<0.001$.

\section{Results}

The age of the patients ranged from 31 to 78 years (median 56 years). The mean value for the ER-EIA assay was $60.0 \mathrm{fmol} / \mathrm{mg}$ protein (median $25 \mathrm{fmol} / \mathrm{mg}$ protein). The immunohistochemical staining with the 1D5 antibody produced uniform nuclear staining (fig 1). The mean $\mathrm{H}$ score for the ER-IHA-1D5 assay was 60.5 (median 48 ).

There was an $86 \%$ concordance between ER-EIA and ER-IHA using the anti-1D5 antibody (table $1 ; \chi^{2}=55.6 ; \mathrm{p}<0.001$ ); $54 \%$ $(64 / 119)$ of the tumours were oestrogen receptor positive and $32 \%$ (38/119) were oestrogen receptor negative by both assays. However, the quantitative results from the ER-EIA and the semiquantitative scores from the ER-IHA do not seem to be linearly related (fig 2).

A mismatch between the EIA and IHA was observed in 17 cases (fig 3 ). Seven of these tumours were EIA positive but IHA positive, although five out of seven had EIA values between 5 and $10 \mathrm{fmol} / \mathrm{mg}$ protein. The other 10 discordant tumours were EIA positive and IHA negative; four of these tumours were completely negative by IHA in the section studied and the EIA values in these four instances were between 10 and $18 \mathrm{fmol} / \mathrm{mg}$ protein, which may also be considered borderline. Half of the remaining six EIA positive IHA negative tumours, however, had EIA values greater than $50 \mathrm{fmol} / \mathrm{mg}$ protein (table 2).

A repeat ER-IHA-1D5 assay on the 17 discordant tumours showed that the $\mathrm{H}$ scores obtained initially were entirely reproducible. Identical sections of tumour were stained in a separate assay with the $\mathrm{H} 222$ antibody. A set of five positive and five negative concordant controls from the original series were also examined by both assays (table 2). As well as straightforward check of the data, this allowed us to address the possibility that some or all of these discrepancies were due to the antibodies targeting different ends of the oestrogen receptor.

In some tumours the IHA staining signal obtained with the 1D5 antibody was of greater intensity than that obtained with the H222 antibody. The results obtained using the two antibodies on the mismatched and control tumours are summarised in table 2 . Excellent agreement was observed with both antibodies on the sets of positive and negative control tumours. Of the 10 tumours which were EIA positive but IHA negative, the $\mathrm{H} 222$ staining agreed with the $1 \mathrm{D} 5$ staining in 


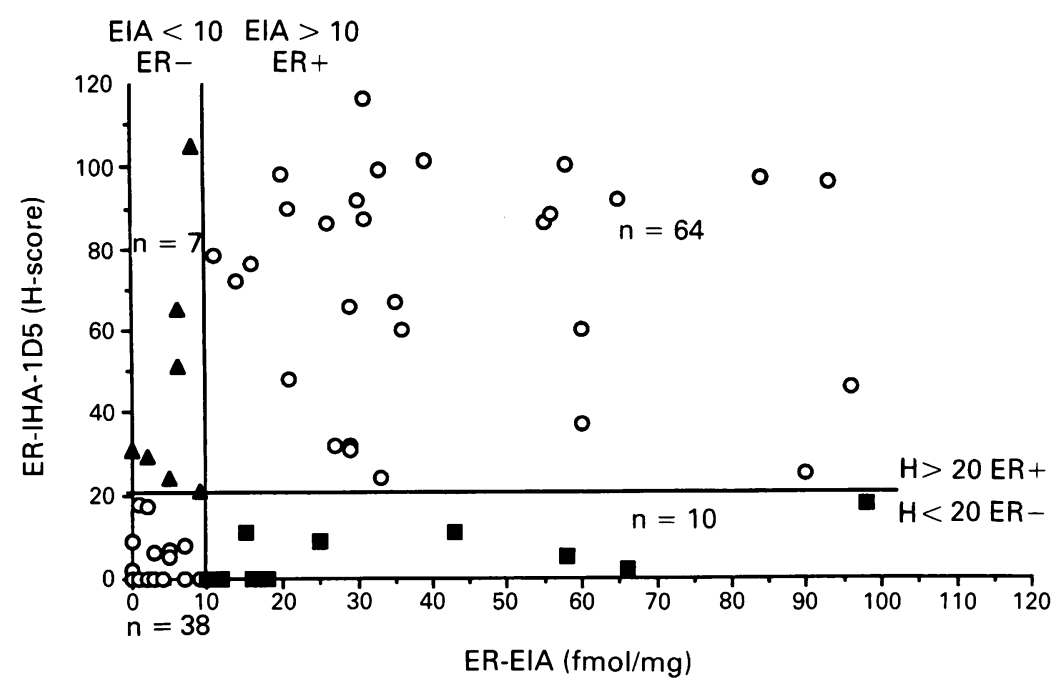

Figure 3 Scattergram for tumours with ELA values of $<120 \mathrm{fmol} / \mathrm{mg}$ protein and $I H A$ scores of <120. Positive cut off for ER-EIA is $10 \mathrm{fmol} / \mathrm{mg}$ protein, and for ER-IHA is $H$ score 20. Discrepancies are shown as follows: $\mathbf{\square}=E I A$ positive/IHA negative cases $(n=10)$;

$\Delta=E I A$ negative/IHA positive cases $(n=7)$.

Table 2 ER-ELA results compared with ER-IHA testing with both $1 D 5$ and $H 222$ antibodies in 17 cases where EIA and IHA originally mismatched

\begin{tabular}{lcc}
\hline ELA Abbott fmol/mg protein & $I H A-1 D 5 H$ score & IHA-H-222 H score \\
\hline ELA negative/IHA-1D5 positive tumour mismatches: & \\
2 & 29 & 12 \\
5 & 24 & 18 \\
9 & 21 & 15 \\
6 & 65 & 1 \\
6 & 51 & 1 \\
0 & 31 & 3 \\
8 & 105 & \\
ELA positive/IHA 1D5 negative tumour mismatches: & 31 \\
98 & 18 & 10 \\
66 & 2 & 18 \\
58 & 5 & 12 \\
43 & 11 & 0 \\
25 & 9 & 0 \\
18 & 0 & 0 \\
16 & 0 (Benign positive) & 5 \\
15 & 11 & 0 \\
12 & 0 & 0 \\
10 & 0 & \\
ER positive control tumours: & & 132 \\
268 & 133 & 124 \\
175 & 150 & 72 \\
146 & 100 & 110 \\
306 & 112 & 185 \\
500 & 185 & \\
ER negative control tumours: & & 0 \\
0 & 0 & 0 \\
1 & 0 & 0 \\
1 & 0 & 0 \\
0 & 0 & 0 \\
1 & 0 &
\end{tabular}

Ten control tumours (five strongly positive and five negative) are included.

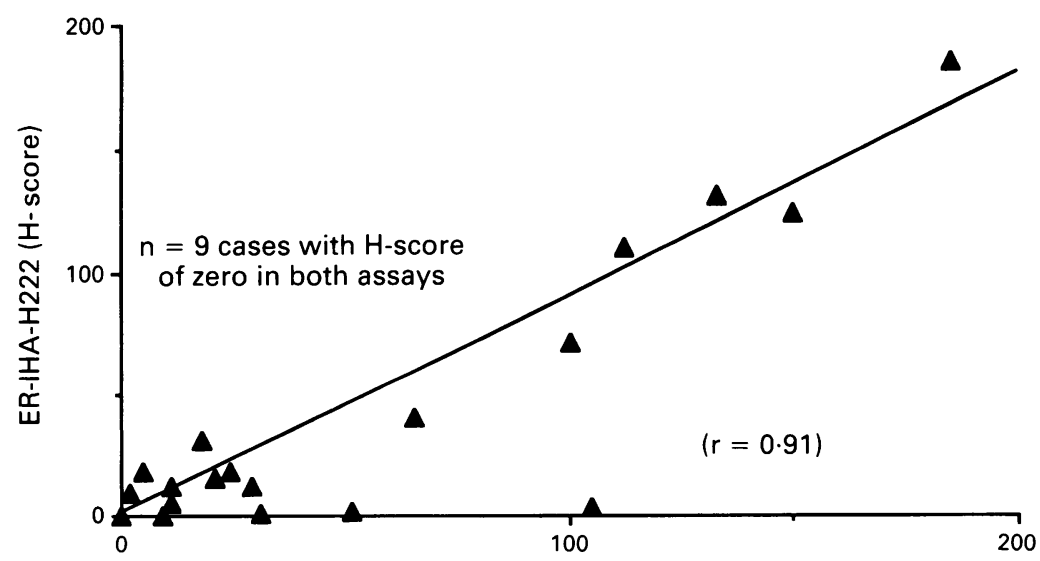

ER-IHA-1D5 (H-score) Figure 4 Correlation between $H$ scores from $E R-I H A-1 D 5$ and $E R-I H A-H 222$ assays in
17 tumours which were discrepant between $E I A$ and IHA-1DS and in five positive and five negative concordant control tumours. nine cases, with four of these scoring zero by both antibodies. In two tumours there was positive staining in benign epithelium but negative staining in adjacent invasive carcinoma cells. This resulted in a positive EIA score in both cases but a negative IHA score. In the one remaining tumour where the antibodies did not agree, the $\mathrm{H} 222$ score was 31 (positive) whereas the 1D5 score was 18 (borderline negative), in a tumour which had an ER value of $98 \mathrm{fmol} / \mathrm{mg}$ protein by EIA.

Of the seven tumours which were EIA positive but IHA positive, the H222-IHA confirmed a positive result in only one case. However, in a further three cases the scores were only borderline positive by $1 \mathrm{D} 5$ and borderline negative by $\mathrm{H} 222$. There remained three tumours which were highly positive by $1 \mathrm{D} 5$ but which remained unequivocally negative by $\mathrm{H} 222$. In these discrepant cases the staining with 1D5 was heterogenous among the invasive carcinoma cells. Overall, there was a linear correlation between the semiquantitative staining obtained with the H222 and 1D5 anti-ER antibodies which approaches unity (fig 4) $(r=0.91 ; p<0.001)$.

\section{Discussion}

Previous studies have shown a good correlation between the ER-ICA method on frozen sections and the established ER-DCC or EREIA assays. ${ }^{1314}$ Overall, the median concordance of the published studies is $86 \% .^{15} \mathrm{~A}$ reliable paraffin wax embedded ER-IHA method would permit retrospective studies on archival material from large cohorts of patients subsequently treated with endocrine treatment in whom data on response, disease free interval, or survival are now known. In many clinical studies on adjuvant endocrine treatment, less than $30 \%$ of patients have oestrogen receptor status available as frozen tissue.

The detection of oestrogen receptor, however, has been hampered by the loss of immunoreactivity during tissue processing of paraffin wax embedded material. Antigen retrieval methods, such as proteolytic enzyme digestion, ${ }^{16}$ together with amplification of the signal with the more sensitive avidin biotin peroxidase complex, ${ }^{17}$ have improved oestrogen receptor detection. Different fixation methods may also determine whether oestrogen receptor can be detected reliably..$^{15}$ Direct comparison of ER-IHA on paraffin wax sections with ER-ICA on frozen sections has shown a concordance of between $82 \%$ and $100 \%$, although these studies suggest that paraffin wax assays are less sensitive. ${ }^{18-21}$ In previously published series comparing ERIHA on paraffin wax embedded material with ER-DCC, the concordance has varied between 65 and $96 \% .{ }^{15}$ There has been only one series comparing paraffin wax assays with ER-EIA which showed a concordance of $95 \%$ in 55 tumours. $^{7}$ In these comparisons with biochemical assays the oestrogen receptor content showed less concordance for tumours with oestrogen receptor values of $<100$ 
$\mathrm{fmol} / \mathrm{mg}$ protein. With the ER-ICA approach there is also considerable variation in the cutoff level quoted in published series for the semiquantification of oestrogen receptor expression in tissue sections, ranging from more than one cell positive to $>30 \%$ cells staining positive. ${ }^{15}$ Clearly, these differing cut offs may explain at least in part the variability in concordance from 68 to $93 \%$ when comparing ER-DCC or ER-EIA with ER-ICA.

In our study we used microwave treatment for antigen retrieval together with a new monoclonal antibody (1D5) directed against the $\mathrm{N}$-terminal of the oestrogen receptor protein. The incidence of oestrogen receptor positivity for this series of 119 primary untreated breast cancers is in line with other published results. Using a cut off of $10 \mathrm{fmol} / \mathrm{mg}$ protein for the ER-EIA, we assessed the validity of the ER-IHA in paraffin wax embedded material with $1 \mathrm{D} 5$ using the $\mathrm{H}$ score method for semiquantitation with a positive cut off of 20 . The concordance rate of $86 \%$ is similar to that in studies comparing biochemical assays with ER-ICA by $\mathrm{H} 222$ on frozen sections.

However, as fig 2 shows, the quantitative correlation between the two methods is not linear. For tumours with ER-EIA values of $>100 \mathrm{fmol} / \mathrm{mg}$ protein, all tissue sections had ER-IHA $\mathrm{H}$ scores of $>80$ which implies excellent concordance between the two techniques for tumours rich in oestrogen receptor. For tumours with less than $100 \mathrm{fmol} / \mathrm{mg}$ on ER-EIA, there was much greater variation in the $\mathrm{H}$ score (fig 3 ). This is probably mainly due to there not being a linear correlation between protein concentration and the intensity of the colour developed.

Using the cut offs described, there were 17 tumours in which the ER-IHA and ER-EIA mismatched. By repeating the assay in these tumours with both 1D5 and H222, four of these tumours remained completely negative by IHA in the section studied. The EIA values in these four tumours was between 10 and 18 $\mathrm{fmol} / \mathrm{mg}$ protein, which is borderline, and in this situation the IHA result may have been due to sampling of an oestrogen receptor negative area. Half of the remaining 6 EIA positive IHA negative tumours had EIA values greater than $50 \mathrm{fmol} / \mathrm{mg}$ protein, and in these tumours the $\mathrm{H} 222$ score was between 10 and 31. Therefore, several of the apparent discrepancies between ER-IHA and ER-EIA may be explained by borderline results. A further potential explanation for these EIA positive IHA negative discrepancies could be oestrogen receptor positive benign elements present in the tumour which in a tumour homogenate would give an EIA positive result.

Three tumours remained discordant following reassessment. These tumours, which had EIA values of 0,6 , and $8 \mathrm{fmol} / \mathrm{mg}$ protein, were strongly positive with 1D5 and negative with the H222 antibody. The ER-EIA technique uses a sandwich method with the D547 antibody (against the central part of the receptor) in the solid phase and the H222 antibody (against the hormone binding domain) labelled to the peroxidase enzyme.
Thus, both the ER-EIA and ER-IHA-H222 assays require an intact hormone binding domain to detect oestrogen receptor protein. There are a substantial number of reports of oestrogen receptor molecular variants which lack one part or other of the full molecule. Receptor with a mutated or absent ligand binding domain might be negative by these two assays, but positive by the ER-IHA-1D5 directed against the $\mathrm{N}$-terminal of the receptor. Such a receptor variant has been suggested as existing following the discovery of variant forms of messenger RNA (mRNA) in human breast cancers in which the exon-5 region, coding for part of the ligand binding domain, has been spliced out during transcription. ${ }^{22}$ These variant forms of mRNA are particularly abundant in the rare tumour phenotype which is ER negative by conventional assay, but which expresses high levels of the oestrogen- dependent protein PgR. It is interesting to note, therefore, that two of the three remaining discordant tumours in our series which are only positive by ER-IHA-1D5 are of the rare ER negative PgR positive phenotype. Thus, this antibody may be of potential use in investigating variant forms of oestrogen receptor protein which are not detected by conventional assays.

In conclusion, the ER-IHA which we have described using the 1D5 antibody shows good concordance with the established biochemical ER-EIA assay. Discrepancies may occur among tumours with borderline oestrogen receptor ER-EIA values. The use of separate monoclonal antibodies directed against different parts of the receptor may also be of value in the investigation of variant forms of oestrogen receptor.

G Saccani Jotti was supported in part by the following grants: CNR AI93.00553.04, CNR ACRO Project, CNR Bilateral 93.00406.CT04, and MURST 40\% (Italy). SRDJ, MD, and SD were all supported by the Cancer Research Campaign.

1 Walker KJ, Bouzubar N, Robertson J, Ellis IO, Elston W, Blamey RW, et al. Immunocytochemical localisation of estrogen receptor in human breast tissue. Cancer Res estrogen receptor

2 DeSombre ER, Thorpe SM, Rose C, Blough RR, Andersen KW, Rasmussen BB, et al. Prognostic usefulness of estrogen receptor immunocytochemical assays for human breast cancer. Cancer Res (Suppl) 1986;46: 4256s-64s.

3 Harland RNL, Barnes DM, Howell A, Ribiero GG, Taylor $\mathrm{J}$, Sellwood RA. Variation of receptor status in cancer of the breast. Br $\mathcal{F}$ Cancer 1983;47:51 1-5.

4 Greene GL, Jensen EV. Monoclonal antibodies as probes for estrogen receptor detection and characterisation. for estrogen receptor detection
f Steroid Biochem 1982;16:353-9.

5 Bojar H. Quality control requirements in estrogen receptor determination. Cancer Res 1986;46:4249s-50s.

6 Thorpe SM. Monoclonal antibody technique for detection of estrogen receptors in human breast cancer. Greater sensitivity and more accurate classification of receptor status than the dextran-coated charcoal method. Cancer Res 1987;47:6572-5.

7 Andersen J, Bentzen SM, Poulsen HS. Relationship between radioligand binding assay, immunoenzyme assay and immunohistochemical assay for estrogen receptors in human breast cancer and association with tumour differentiation. Eur f Cancer 1988;24:377-84.

8 Miller RT, Hapke MR, Greene GL. Immunocytochemical assay for estrogen receptor with monoclonal antibody D753P $\gamma$ in routinely processed formaldehyde-fixed breast tissue. Cancer 1993;71:3541-6.

9 DeRosa CM, Ozzello L, Greene GL, Habif DV. Immunostaining of estrogen receptor in paraffin sections of breast carcinomas using monoclonal antibody D75P3gamma: effects of fixation. Am F Surg Pathol

10 Allred CD. Should immunohistochemical examination 
replace biochemical hormone receptor assays in breast cancer? Am f Clin Pathol 1993;46:1-3.

11 Al Saati T, Clamens S, Cohen-Knafo E, Faye JC, Prats H, Comdre JM, et al. Production of monoclonal antibodies to human oestrogen receptor protein (ER) using recombinant ER (RER). Int $f$ Cancer 1993;55:651-4.

12 Andersen J, Orntoft TF, Poulsen HS. Immunohistochemical demonstration of estrogen receptors (ER) in formalin-fixed, paraffin-embedded human breast in formalin-fixed, paraffin-embedded human breast $\Im$ Histochem Cytochem 1988;36:1553-60.

13 Heubner A, Beck T, Grill H-J, Pollow K. Comparison of immunocytochemical estrogen receptor assay, estrogen receptor enzyme immuno-assay, and radio-ligand estrogen receptor assay in human breast cancer and uterine tissue. Cancer Res 1986;46:4191-5.

14 Hanna W, Mobbs BG. Comparative evaluation of ER-ICA and enzyme immunoassay for the quantification of estrogen receptors in breast cancer. Am $¥$ Clin Pathol gen receptors

15 Andersen J. Determination of estrogen receptors in paraffinembedded tissue. Acta Oncologica 1992;31:611-27.

16 Curran RC, Gregory J. The unmasking of antigens on paraffin sections of tissue by trypsin. Experimenta 1977 33:1400-1

17 Hsu S-M, Raine L, Fanger H. Use of avidin-biotin- peroxidase complex (ABC) in immunoperoxidase techniques: $A$ comparison between $\mathrm{ABC}$ and unlabelled antibody (PAP) procedures. $\mathcal{f}$ Histochem Cytochem 1981; 29:577-80.

18 Anderson J, Thorpe SM, King WJ, Rose C, Christensen I, Rasmussen BB, et al. The prognostic value of immunohistochemical estrogen receptor analysis in paraffinhistochemical estrogen receptor analysis in paraffin-
embedded and frozen sections versus that of steroid embedded and frozen sections versus that

19 Shimada A, Kimura S, Abe K, Nagasaki K, Adachi I, Yamaguchi $\mathrm{K}$, et al. Immunocytochemical staining of estrogen receptor in paraffin sections of human breast cancer by use of monoclonal antibody: Comparison with that in frozen sections. Proc Natl Acad Sci USA $1985 ; 82: 4803-7$

20 Shintaku P, Said JW. Detection of estrogen receptors with monoclonal antibodies in routinely processed formalinfixed paraffin sections of breast carcinoma. Am $\Im$ Pathol 1987;87:161-7.

21 Hiort O, Kwan WL, DeLellis RA. Immunohistochemistry of oestrogen receptor protein in paraffin sections. Am $\mathcal{F}$ Clin Pathol 1988;90:559-63.

22 Fuqua SAW, Fitzgerald SD, Chamness GC, Tandon AK, McDonnell DP, Nawaz Z, et al. Variant human breast tumour estrogen receptor with constitutive transcriptional activity. Cancer Res 1991;51:105-9. 